\title{
Active Ingredients Combinations for Pathogens Control on Cucumber Crops under High Plastic Tunnels
}

\author{
Gabriela ȘOVĂREL*, Marcel COSTACHE, Ana - Emilia CENUȘĂ \\ Plant protection laboratory, Research - Development Institute for Vegetable and Flower Growing Vidra, \\ Ilfov, Romania \\ *)Corresponding author, e-mail: gabriela_sovarel@yahoo.com
}

BulletinUASVM Horticulture 74(1) / 2017

Print ISSN 1843-5254, Electronic ISSN 1843-5394

DOI:10.15835/buasvmcn-hort:12309

\begin{abstract}
In Romania, Pseudoperonospora cubensis and Sphaerotheca fuliginea are the most important pathogens on cucumber crops under high plastic tunnels. For controlling these pathogens there are applied products with different active ingredients: dimethomorph $9 \%$, mancozeb $60 \%$, myclobutanil $240 \mathrm{~g} / \mathrm{l}$, penconazole $100 \mathrm{~g} / \mathrm{l}$, iprovalicarb $8.4 \%$, copper oxychloride $40 \%$. The best efficacy for controlling Pseudoperonospora cubensis and Sphaerotheca fuliginea was obtained both combinations foseyl-aluminium $80 \% 0.2 \%+$ penconazole $100 \mathrm{~g} / \mathrm{l}$ $0.025 \%(84.8 \% .100 \%)$ and fosetyl aluminium $80 \% 0.2 \%$ + myclobutanil $240 \mathrm{~g} / \mathrm{l} 0.02 \%$ (83.7\%, 100\%).
\end{abstract}

Keywords: cucumber, pathogens control, plastic tunnels

\section{Introduction}

In Romania, Pseudoperonospora cubensis and Sphaerotheca fuliginea are the most important pathogens on cucumber crops under high plastic tunnels (Costache et al. 2007). A study realised in Bulgaria, in the period 2006 - 2011 showed that the combination with active ingredients (dimethomorph 225g/l + initium $300 \mathrm{~g} / \mathrm{l}$ ) 0.07\%, cymoxanil $60 \mathrm{~g} / \mathrm{kg}+$ copper hydroxide $436 \mathrm{~g} / \mathrm{kg}$ ) $0.2 \%$ and trifloxistrobin $25 \mathrm{~g} / \mathrm{kg}+$ cymoxanil 25 $\mathrm{g} / \mathrm{kg}) \quad 0.05 \%$ had good efficacy $(86.13-89.31 \%)$ for controlling Pseudoperonospora cubensis on cucumbers (Masheva et al. 2013). Famoxadone + cymoxanil was the most effective treatment against downy mildew followed by clove oil, zinc oxide nanoparticles, EMs1, B. pumillus, B. subtilus and castor oil, respectively in two growing seasons (2012-2013/2013-2014; Mohamed et al. 2016),

\section{Aims and objectives}

The aim of this research is to identify active ingredients combinations for simultaneously control of these pathogens.

\section{Materials and methods}

The research take place during 2015, under high plastic tunnels with multiple possibility of ventilation, using cucumber hybrid Mirabelle $\mathrm{F}_{1}$. Planting date august 1,2015 , density 33000 plants/ha.

For controlling Pseudoperonospora cubensis and Sphaerotheca fuliginea were used 7 vatriants: 1. (dimethomorph $9 \%+$ mancozeb $60 \%$ ) $0.2 \%$ + myclobutanil $240 \mathrm{~g} / \mathrm{l}$ 0.02\%; 2. (dimethomorph $9 \%+$ mancozeb $60 \%) 0.2 \%$ + penconazole 100 g/l $0.025 \%$; 3. fosetyl-aluminium $80 \% 0.2 \%+$ myclobutanil $240 \mathrm{~g} / \mathrm{l} 0.02 \%$; 4 . fosetyl-aluminium $80 \% 0.2 \%+$ penconazole $100 \mathrm{~g} / \mathrm{l} \quad 0.025 \%$; 5. (iprovalicarb $8.4 \%+$ copper oxychloride $40 \%) 0.2 \%$ + myclobutanil $240 \mathrm{~g} / \mathrm{l}$ 0.02\%; 6 . (iprovalicarb 8,4 \% + copper oxychloride 40 \%) $0.2 \%$ + penconazole $100 \mathrm{~g} / \mathrm{l} \quad 0.025 \%$ and 7 . Untreated check.

Were made three applications during vegetable period. First application was made at beginning infection risk (02.09) and the others at 7 days intervals (09.09 and 16.09). 
Tab. 1. Efficacy of some fungicides combinations for simultaneously control of Pseudoperonospora cubensis and Sphaerotheca fuliginea pathogens on cucumbers

\begin{tabular}{cccccccccc}
\hline \multirow{2}{*}{ Variant } & $\begin{array}{c}\text { Concentration } \\
\text { (\%) }\end{array}$ & \multicolumn{2}{c}{ Degree of attack (\%) } & \multicolumn{2}{c}{ Efficacy (\%) } & \multicolumn{3}{c}{ Yield } \\
\cline { 3 - 10 } & P.cubensis & S.fuliginea & Total & P.cubensis & S.fuliginea & Total & t/ha & $\%$ \\
\hline 1 & $0.2+0.02$ & 16.9 & 0 & 16.9 & 73.0 & 100.0 & 82.8 & 38.6 & 112.9 \\
\hline 2 & $0.2+0.025$ & 18.5 & 0 & 18.5 & 70.4 & 100.0 & 81.2 & 38.4 & 112.3 \\
\hline 3 & $0.2+0.02$ & 10.2 & 0 & 10.2 & 83.7 & 100.0 & 89.6 & 39.3 & 114.9 \\
\hline 4 & $0.2+0.025$ & 9.5 & 0 & 9.5 & 84.8 & 100.0 & 90.3 & 39.7 & 116.1 \\
\hline 5 & $0.2+0.02$ & 16.0 & 0 & 16.0 & 74.4 & 100.0 & 83.7 & 39.0 & 114.0 \\
\hline 6 & $0.2+0.025$ & 16.8 & 0.2 & 17.0 & 73.1 & 99.4 & 82.7 & 38.7 & 113.1 \\
\hline 7 & - & 62.5 & 35.8 & 98.3 & - & - & - & 34.2 & 100.0 \\
\hline
\end{tabular}

There were made observations regarding the frequency (\%) and severity (\%) of pathogen attack and were calculated the attack degree (\%) and efficacy (\%). The degree of attack was calculated with formula (F\% x S \%) /100 and efficacy with formula (DA untreated \% - DA treated \%) x 100/ DA untreated.

\section{Results and discussion}

The best efficacy for controlling Pseudoperonospora cubensis and Sphaerotheca fuliginea was obtained both variants 4 . fosetyl-aluminium $80 \%$ $0.2 \%$ + penconazole $100 \mathrm{~g} / \mathrm{l} \quad 0.025 \%$ (84.8\%. $100 \%$ ) and 3. fosetyl-aluminium 80\% $0.2 \%+$ myclobutanil $240 \mathrm{~g} / \mathrm{l}$ 0.02\% (83.7\%, 100\%).

The degree of attack on untreated check was $62.5 \%$ at Pseudoperonospora cubensis and $35.8 \%$ at Sphaerotheca fuliginea.

The actives substances myclobutanil $240 \mathrm{~g} / \mathrm{l}$ and penconazole $100 \mathrm{~g} / \mathrm{l}$, applied for controlling Sphaerotheca fuliginea have a very good efficacy.

\section{Conclusion}

There were identified several product combinations which provide a good control of main pathogens on cucumbers under protected crops.

The best efficacy for controlling Pseudoperonospora cubensis and Sphaerotheca fuliginea was obtained both fosetyl-aluminium $80 \% 0.2 \%$ + penconazole $100 \mathrm{~g} / \mathrm{l} 0.025 \%$ and fosetyl-aluminium $80 \%$ 0.2\% + myclobutanil $240 \mathrm{~g} / \mathrm{l}$ 0.02\%.

\section{REFERENCES}

1. Costache M, Roman T, Costache C (2007). Bolile si daunatorii culturilor de legume. Editura Agris, Bucuresti.

2. Masheva S, Velcov N, Valchev N, Yarkova V.(2013). Screening of plant protection products against downy mildew on cucumbers (Pseudoperonospora cubensis) in cultivation facilities. Agricultural and Science Technology. ISSN 1314-412x, vol. 5, no. 2, pg. 194-199.

3. Mohamed A, Hamza A, Derbalah A (2016). Recent approaches for controlling downy mildew of cucumber under greenhouse conditions. Plant Protect. Sci., vol. 52, no.1.pg. 1-9. 